\title{
Adaptive correction of depth-induced aberrations in multiphoton scanning microscopy using a deformable mirror
}

\author{
L. SHERMAN*, J. Y. YE*, O. ALBERT† \& T. B. NORRIS* \\ *Center for Ultrafast Optical Science, 1006 IST Bldg, University of Michigan, 2200 Bonisteel Blvd, \\ Ann Arbor, MI 48109, U.S.A. \\ †Laboratoire d'Optique Appliquee - ENSTA, Chemin de la Huniere, 91761 Palaiseau Cedex, France
}

Key words. Adaptive aberration correction, confocal microscopy, depth-induced aberration, spherical aberration.

\section{Summary}

We demonstrate adaptive aberration correction for depthinduced spherical aberration in a multiphoton scanning microscope with a micromachined deformable mirror. Correction was made using a genetic learning algorithm with two-photon fluorescence intensity feedback to determine the desired shape for an adaptive mirror. For a 40×/0.6 NA long working distance objective, the axial scanning range was increased from $150 \mathrm{~mm}$ to $600 \mathrm{~mm}$.

\section{Introduction}

The elegant central idea of confocal microscopy is that it effectively images only the region of the specimen that lies very near the focal plane. This optical sectioning allows one to image a series of through-focus and off-axis points that can be processed to a three-dimensional (3D) volume; multiphoton scanning microscopy allows for confocal imaging in the absence of an aperture (Denk et al., 1990). Aberrations present in a 3D confocal microscope system ultimately limit the volume that can be imaged. In the lateral scanning direction, aberrations are present due to beam scanning, i.e. sending the beam at an angle through the objective to achieve lateral translation in the focal plane. In the longitudinal direction the use of high numerical aperture (NA) objectives immersed in a fluid that is not index matched to the specimen gives rise to spherical aberration. In this case, as the confocal microscope focuses deeper into the sample, the axial resolution degrades and signal levels drop. The effects of aberrations in the system are exacerbated when imaging with multiphoton processes such as two-photon fluorescence or third harmonic generation, where the signal levels have a quadratic or cubic intensity dependence of the incident light, respectively.

Correspondence: T. Norris. Tel.: +1 734763 4875; fax: +1 734763 4976; e-mail: tnorris@eecs.umich.edu
Much work has been done analysing the effects of imaging with a high NA objective into a specimen (Hell et al., 1993; Török et al., 1997). Several methods have been suggested to overcome this decrease in signal and resolution, such as inserting weak aberration correction lenses, or altering the tube length of the objective (Sheppard \& Gu, 1991). The latter employed a static correction that worked to restore much of the resolution at a certain depth, but cannot be easily adjusted for varying depths. Hellmuth et al. (1988) showed that if one overcorrects for the cover glass thickness, or uses a less than nominal cover glass thickness, it would compensate for spherical aberration at a certain depth in a specimen with index mismatch. Wan et al. (2000) changed the immersion medium to do the same. Booth \& Wilson (2000) used both of these methods to compensate for spherical aberration in skin as well as employing an iris to reduce the pupil area. All of the methods discussed use static means to correct for specimen-induced spherical aberration at a specific depth, not a dynamic means of compensation as a beam is scanned deeper into a sample.

With recent advances in adaptive aberration correction it is now possible to employ dynamic compensation of aberrations, which can be applied during the scanning process for a continuous volume of aberration free imaging. Neil et al. (2000) have demonstrated adaptive correction by first measuring the aberrations of the beam at the focus with a modal wavefront sensor and then applying the phase conjugate of the measured aberrations with a liquid crystal spatial light modulator. This method follows the traditional method of adaptive aberration correction first used in astronomy (Tyson, 1991). However, there is significant loss of power due to both the continuous monitoring of the wavefront, and the liquid crystal modulator.

In this paper we show a technique for dynamic specimeninduced spherical aberration correction without the use of a wavefront measurement and the subsequent loss of power. A deformable mirror is used for the dynamic wavefront correction with a genetic algorithm (GA) that maximizes the two-photon 
signal from the sample to determine the appropriate wavefront. This method was first used for adaptive correction of offaxis aberrations (Albert et al., 2000). Together, the dynamic correction for depth-induced spherical aberration and off-axis aberrations can provide for full adaptive aberration compensation over a $3 \mathrm{D}$ volume.

\section{The aberrated confocal point spread function}

The two-photon fluorescence intensity depends on the threedimensional light distribution near the focus of the fundamental excitation beam. The image is described by the magnification of the lens and the Fresnel diffraction from the lens (Eq. (1)), where $J_{0}$ is a zero-order Bessel function. Effects of the imaging system, such as aberrations, can be described by the phase aberration function $\Phi(d, \rho)$, where $d$ represents the depth and $\rho$ the normalized radial coordinate in the plane of the pupil (Wilson \& Sheppard, 1984).

$$
I(\rho, z)=\left|K \int_{-d}^{\infty} \exp \left(-\imath k_{1} \Phi(d, \rho)\right) \exp \left(-\frac{\imath u \rho}{2}\right) J_{0}(\rho v) \rho d \rho\right|^{4}
$$

where $\rho=r^{\prime} / a$, and $r^{\prime}$ is the radial coordinate in the pupil plane of the microscope objective, and $a$ the aperture of the objective. The normalized radial component in the image plane is $v=\frac{2 \pi n a r}{\lambda f}$, where $r$ is the radial distance and $f$ is the focal length. The normalized longitudinal coordinate $u$ is related to the distance along the optical axis, $z$, by $u=\frac{8 \pi n z}{\lambda} \sin ^{2} \frac{\alpha_{1}}{2}$.

The aberration due to depth scanning arises from the refractive-index mismatch between the immersion medium of the objective and the specimen. The phase aberration, $\Phi(d, \rho)$, due to focusing a depth, $d$, into refractive index mismatched media, is described as (Booth et al., 1998):

$$
\Phi(d, \rho)=d\left(n_{2} \cos \phi_{2}-n_{1} \cos \phi_{1}\right)
$$

The phase aberration function is normalized to the aperture where $\rho=\frac{r^{\prime}}{a}=\frac{\sin \phi_{1}}{\sin \alpha_{1}}$, where $\alpha_{1}$ is the maximum angle the rays can travel in medium 1 , defined by the numerical aperture (NA) of the objective where $n_{1} \sin \alpha_{1}=n_{2} \sin \alpha_{2}=$ NA. This leads to a normalized aberration function:

$$
\Phi(d, \rho)=d n_{1} \sin \alpha_{1}\left[\sqrt{\csc ^{2} \alpha_{1}-\rho^{2}}-\sqrt{\csc ^{2} \alpha_{2}-\rho^{2}}\right]
$$

Knowing the aberration function from Eq. (3), it is possible to consider the wavefront shape necessary to compensate for the aberrations due to depth. Török et al. (1995) matched the aberrations due to index of refraction mismatch to Zernike polynomials and obtained an analytical expression for the coefficients $A_{n m}$.

$$
\begin{aligned}
\Phi(d, \rho) & =d n_{1} \sin \alpha_{1}\left[\sqrt{\csc ^{2} \alpha_{1}-\rho^{2}}-\sqrt{\csc ^{2} \alpha_{2}-\rho^{2}}\right] \\
& =A_{00}+\sum_{n=2}^{8} A_{n 0} Z_{n 0}(\rho)
\end{aligned}
$$

Due to the longitudinal nature of the depth-induced aberration only the $m=0$ frequency is considered. Up to $n$ $=8$ must be included for a full match of $\Phi$; however, it has been found that significant improvement in the confocal point spread function (PSF) can already be obtained with compensation of $n=4$ or first order spherical aberration (Booth et al., 1998). Thus, the dynamic aberration correction system must be able to create a $\rho^{4}$ wavefront shape to achieve depth-induced spherical aberration correction.

\section{The experimental system}

Our multiphoton microscopy system is shown in Fig. 1. A $80 \mathrm{MHz}, 10 \mathrm{fs}$ Ti:sapphire oscillator centred at $795 \mathrm{~nm}$ is used for the multiphoton excitation, as short pulses maximize the non-linear excitation for a fixed average power. A prism pair is included for pre-compensation of dispersion due to the microscope objective, to ensure that the laser pulses focused in the sample are optimized for efficient multiphoton excitation. A jet of IR 140 (Exiton, Inc., Dayton, OH, U.S.A.) dye is also incorporated as a saturable absorber to block retro-reflected light into the laser, which is lightscattered or reflected off the sample or cover slip that follows the laser path back to the laser cavity that interrupts the mode-locking of the oscillator. Feedback is especially a problem in third harmonic surface generation with a well-focused beam, and must be controlled to have an effective third harmonic generation imaging system.

The microscope system in this work uses a dry $40 \times / 0.6 \mathrm{NA}$ infinity corrected Olympus long working distance objective designed to look deep into samples such as tissues. For simplicity we use a Coumarin water solution in place of the biological sample that is commonly analysed with multiphoton fluorescence microscopy. The long working distance objective allows one to scan from the surface of the cover glass up to $2 \mathrm{~mm}$ into the sample. Two-photon fluorescence is collected with the objective, passes through a dichroic mirror and a short pass filter, is imaged through a confocal aperture to reduce out-of-focus background and measured with a photomultiplier tube (PMT), or is imaged onto a CCD camera. A photodiode is used to monitor the transmitted fundamental light through the sample for normalization of the two-photon fluorescence.

The main element of the adaptive aberration correction system consists of a silver-coated micromachined deformable mirror (DM) (OKO Technologies, the Netherlands). The DM is $19 \mathrm{~mm}$ in diameter, has 37 electrostatic actuators and a maximum deflection of $3 \mathrm{~mm}$ (Vdovin et al., 1997). The laser is first up-collimated to match the laser to the DM with a $4 f$ telescope, and down-collimated with another $4 f$ system designed to directly image the face of the DM to the entrance pupil of the microscope objective. Thus, the wavefront change introduced by the deformation of the DM will correspond to a wavefront 
Fig. 1. The experimental system consisting of the 10 fs oscillator with dispersion compensation, the adaptive correction system including the deformable mirror, and the confocal microscope with long working distance $40 \times$ air immersion objective.

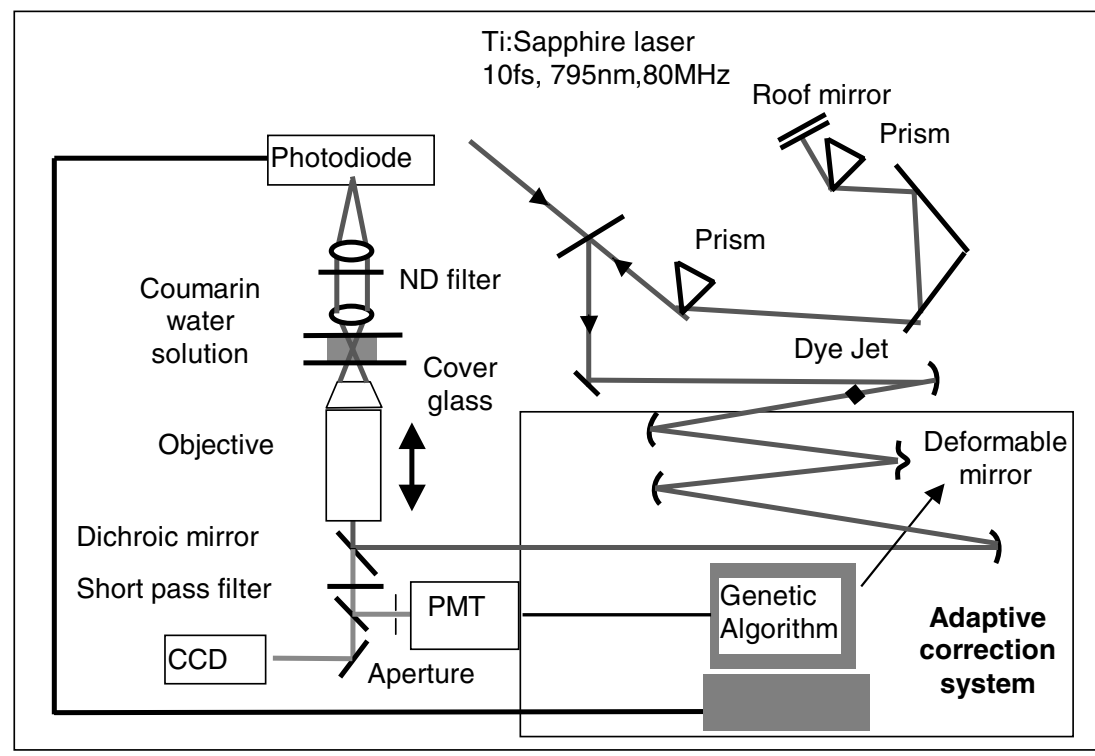

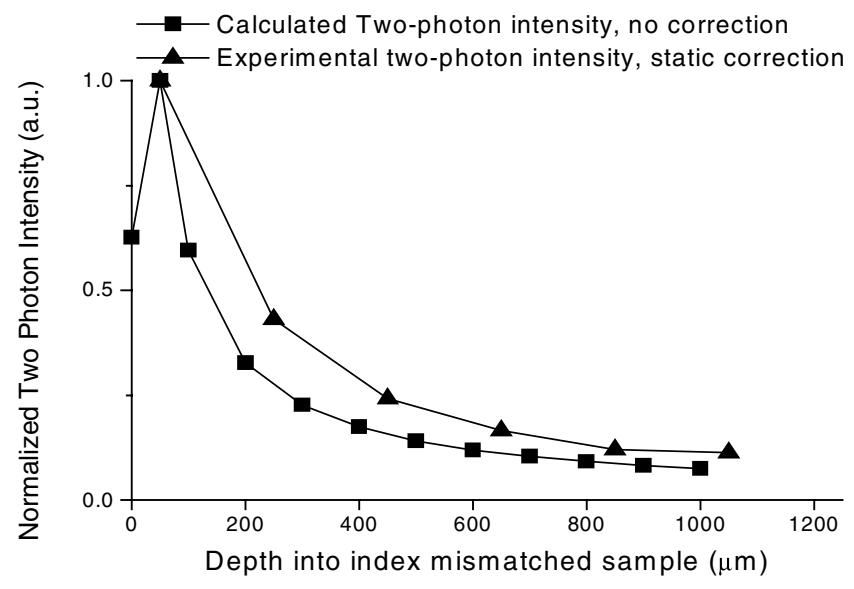

Fig. 2. Two-photon intensity with 0.6 NA Olympus long working distance objective. As the focus is scanned deeper into sample, no correction for depth-induced spherical aberration correction is made.

change at the pupil of the objective without introducing amplitude modulation. We use all reflective optics, except in the objective, to limit pulse broadening due to dispersion. This adds a small amount of astigmatism to the beam. This astigmatism and other small aberrations due to misalignment can be corrected with the DM in the form of a static correction, made when the focus is close to the surface of the sample and no depth-induced spherical aberration is present. However, if the static aberrations are severe, the dynamic range of purely spherical aberration correction will decrease, as the DM has a finite range of travel. Figure 2 shows the calculated two-photon fluorescence intensity for a NA $=0.6$ microscope objective according to Eq. (4), and the experimental two-photon intensity as a function of focus depth with no correction.

\section{Compensation of depth aberrations}

Instead of the traditional approach in adaptive optics of measuring the wavefront and calculating the correction needed, we use a genetic algorithm (GA) with two-photon fluorescence as feedback to determine the optimum shape for the mirror to compensate for any aberrations in the system. This eliminates the need for a formal wavefront measurement because the two-photon signal will increase due to tighter focusing.

The first step in adaptively correcting for depth-induced aberrations is to confirm that the DM can achieve the shape necessary for correction. To verify that the mirror can indeed create a $\rho^{4}$ wavefront shape, we used a three-wave lateral shearing interferometer to measure the wavefront and used that as feedback to the GA (Primot \& Sogno, 1995). We were able to successfully match the $\rho^{4}$ wavefront for up to seven waves of positive aberration according to the mean-square deformation, $\left(\Delta \Phi_{p}\right)^{2}$, of the wavefront as specified in Eq. (5), and plotted in Fig. 3 (Born \& Wolf, 1980).

$$
\left(\Delta \Phi_{p}\right)^{2}=\frac{\int_{0}^{12 \pi} \int_{0}\left(\Phi_{p}-\bar{\Phi}_{p}\right)^{2} \rho d \rho d \theta}{\int_{0}^{12 \pi} \int_{0} \rho d \rho d \theta}
$$

The wavefront, $\Phi_{p}$, is taken for both primary spherical aberration $\left(\rho^{4}\right)$ and the wavefront measured from the DM, and compares the mean square deformation of each case for increasing coefficients. The DM can match the primary spherical aberration very well for coefficients less than six waves, and starts to fall off due to the finite dynamic range of the DM, which is a result of the physical limitations of the deflection of the membrane.

Because of the electrostatic nature of the DM, it can only match the required shape in one direction; essentially the 


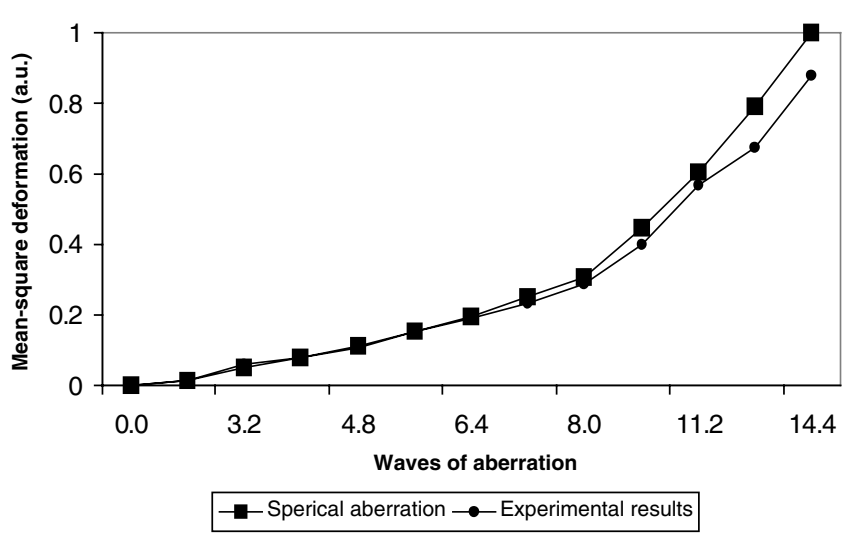

Fig. 3. Mean square deformation of the wavefront for DM fit to $\rho^{4}$ wavefront shape, as measured by a three-wave lateral shearing interferometer.

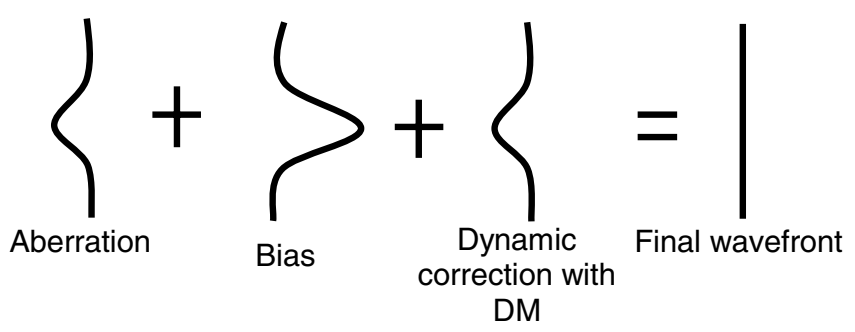

Fig. 4. Schematic diagram of dynamic wavefront compensation with a stationary bias.

actuators can only pull the mirror, not push. This limitation can be overcome by first applying a bias wavefront on the system. Zhu et al. (1999) achieved flexible wavefront generation of Zernike polynomials with the DM, in particular defocus-corrected spherical aberration: $\sqrt{5}\left(6 \rho^{4}-6 \rho^{2}+1\right)$, with judicious application of wavefront bias. Unfortunately, the wavefront correction needed to compensate for the spherical aberration in our set-up, with the microscope objective immersed in air and focusing into a dye-water solution, is in the direction that the DM cannot move. Thus, the method of applying a stationary bias in addition to the DM must be employed to achieve continuous dynamic aberration correction as the focus is scanned deeper into the sample. This is shown schematically in Fig. 4.

The dynamic aberration correction for spherical aberration correction in a biological sample can be made by two different biasing methods, each with its own advantages. The first, and simplest, method is to apply a large negative spherical aberration bias using some optical element in the system, and use the DM to subtract dynamically any unneeded, or residual, negative spherical aberration with a positive displacement. The stationary bias is applied by overcorrecting for the cover glass thickness, either by using a thinner cover glass than is specified, or using an adjustable collar on the microscope designed for variable cover glass thickness.
With the bias applied, but no correction from the mirror, the resolution is decreased at shallow depths close to the cover glass. Instead, the resolution is optimum at some point deeper in the sample due to the applied wavefront bias; in other words, the negative spherical aberration from focusing deeper into the sample matches the positive aberration due to the bias. At larger depths the resolution will drop again, due to the increasing negative spherical aberration from focusing deep into an index-mismatched specimen. This is shown in Fig. 5, where the normalized two-photon fluorescence intensity for a $\mathrm{NA}=0.6$ objective is calculated for normal operation, i.e. no bias conditions or correction, and is plotted next to the experimentally obtained two-photon fluorescence intensity with a negative bias.

Comparison of the calculated two-photon fluorescence intensity with primary spherical aberration removed and the actual experimental system including the DM shows good agreement up to that intermediate depth where the initial stationary bias is overcome. The limitation in range is due to the finite dynamic range of the DM. The micromachined membrane is suspended above the voltage actuators by a distance of $3 \mathrm{~mm}$, and that is the maximum translation. One should note that the calculated values for two-photon fluorescence intensity with primary spherical aberration correction do decrease at extreme depths; this is because higher order spherical aberrations come into play. The DM cannot easily correct for any higher order aberration because the membrane structure can only be pulled; thus, it is not able to form 'ringing' modes as for higher order spherical aberration. Higher order modes are possible with biasing techniques; however, such biasing would limit the range of the modes that could be compensated.

Dynamic aberration is achieved by running the GA to determine the DM wavefront that would best maximize the twophoton intensity at the focus as measured by the fluorescence power. The GA is an evolutionary learning algorithm that starts with a set of random solutions for the DM wavefront as first 'parents'. The random solution is revised by mating the solutions together to obtain a larger set of 'offspring'. These offspring are then tested on the system and the ones that produce the largest two-photon fluorescence intensity at focus are saved to provide the parents for the next generation of offspring. This continues until the two-photon intensity reaches a steady maximum value. This is reiterated for steps of deeper focusing within the specimen. An optimum DM solution will emerge in approximately 10 generations (approximately $3 \mathrm{~min}$, and can be saved for future use, as long as the objective is the same and the location of the DM in relation to the microscope has not been changed. (Further details of the GA implementation may be found in Albert et al., 2000).

By applying a large negative spherical aberration bias the best possible resolution is extended from $50 \mathrm{~mm}$ to $350 \mathrm{~mm}$, and the full width at half maximum (FWHM) of the scanning range is increased from $150 \mathrm{~mm}$ to $600 \mathrm{~mm}$. This is achieved 


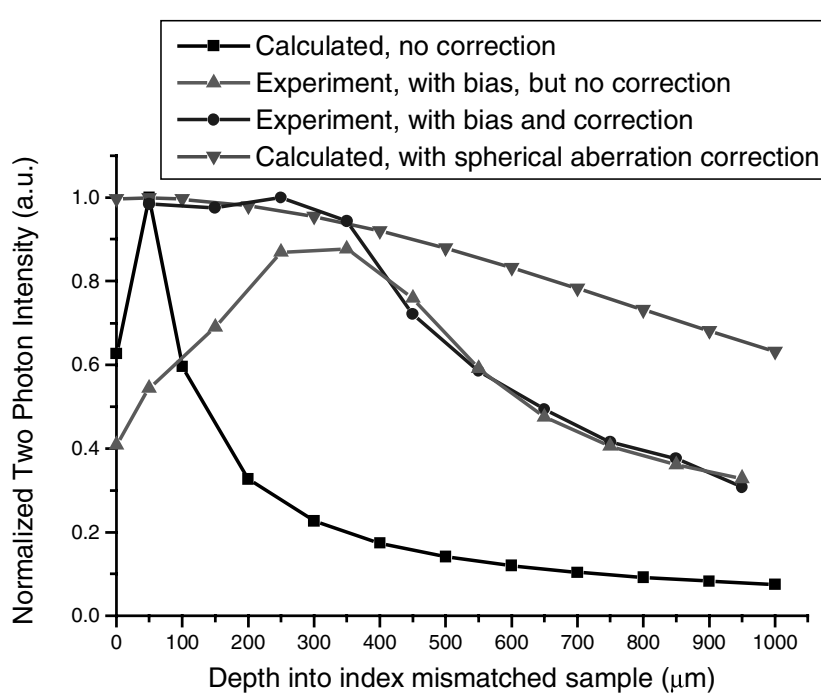

Fig. 5. Calculated two-photon intensity for no correction and correction of primary spherical aberration for NA $=0.6$, and experimental twophoton intensity for the case with negative spherical aberration bias applied with and without dynamic correction with DM.

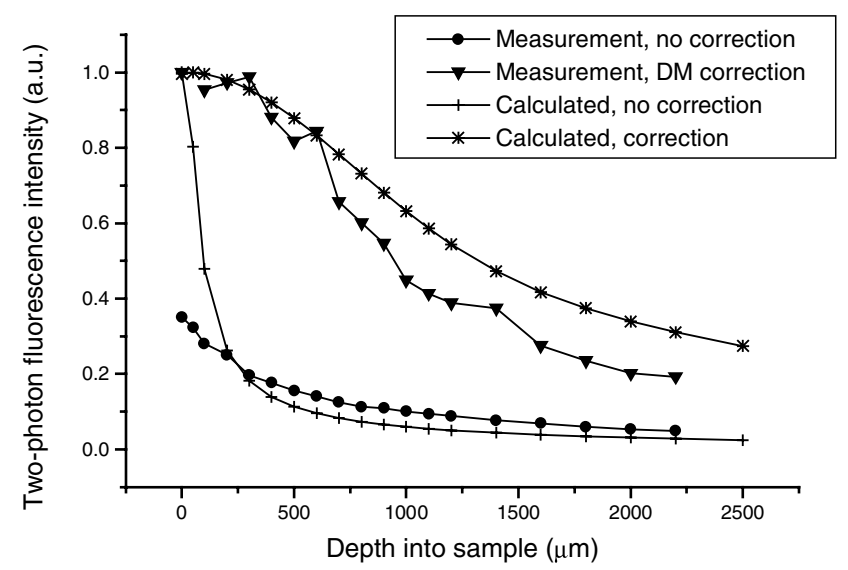

Fig. 6. Normalized two-photon intensity with a defocus bias. very easily by over-correcting for the cover glass thickness and applying a database of DM shapes over the extended range, and with negligible loss in energy due to insertion of the correction system.

The second method of bias application involves using not a spherical aberration bias, but a defocus bias or $\rho^{2}$. One of the lenses in the telescope that images the DM to the microscope objective is translated on-axis, changing the divergence of the incoming beam into the microscope objective from collimated, for an infinity-corrected objective, to diverging out. The DM is then optimized to find the best possible shape to correct for the wavefront error at the entrance to the objective. Figure 6 shows the normalized experimental results for this method plotted next to the calculated intensity levels with and without correction.

Using a defocus bias over a spherical aberration bias does allow for increased scanning range, from $150 \mathrm{~mm}$ to $800 \mathrm{~mm}$, and closer agreement to the calculated possible correction. However, in this scheme energy is lost due to the initial divergence necessary for the bias, as the beam size is expanded at the objective and overfills the pupil substantially. Also, careful monitoring of the laser transmitted through the objective is essential to ensure that the GA results in spherical aberration correction, as opposed to simply increasing the laser energy entering the microscope. This loss of energy, over $50 \%$, is not acceptable for most multiphoton microscopy; however, it may be tolerable for single photon processes.

We next confirmed that the use of the DM to maximize the two-photon fluorescence does indeed increase the imaging resolution. The improved resolution in a CCD image of the two-photon fluorescence at $600 \mathrm{~mm}$ is shown in Fig. 7. For the case of spherical aberration bias, the longitudinal PSF was measured with third harmonic generation (THG). THG is a process that occurs only at the interface where there is some index of refraction discontinuity at regions of high intensity, thus the THG signal will only arise when there is an interface with index of refraction difference at the focus of the laser (Tsang, 1995). For this measurement a quartz plate was
Fig. 7. Normalized CCD image of two-photon fluorescence at a focus depth of $600 \mathrm{~mm}$ with and without correction with the DM.

$2.5 \mu \mathrm{m}$

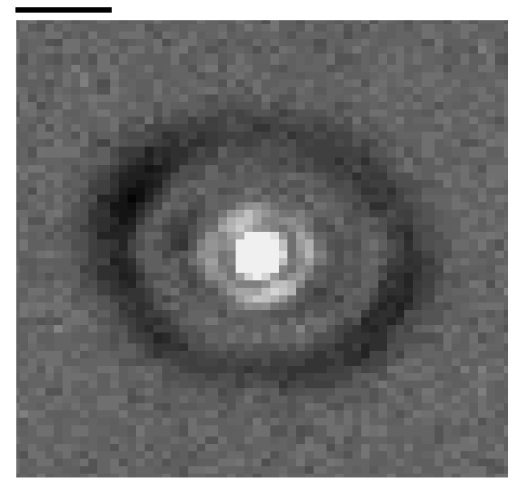

Before correction

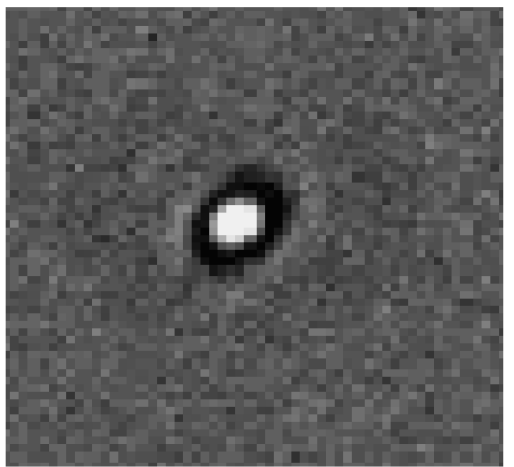

After correction 


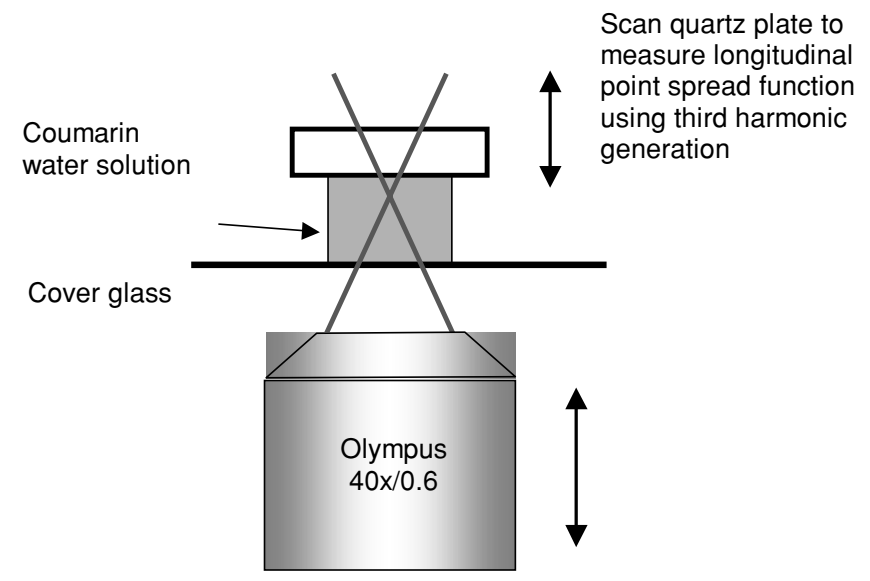

Fig. 8. A quartz plate is scanned through the focus and the third harmonic generation from the surface is measured to record the longitudinal PSF.

scanned from one side of the focus to the other. The THG from the interface between the quartz and the Coumarin water solution was separated from the fundamental light with a prism and detected with a photon counter (see Fig. 8). The normalized longitudinal PSFs clearly indicate an increase of resolution with correction. An example, Fig. 9, shows (at a focus depth of $350 \mathrm{~mm}$ ) near-diffraction-limited FWHM of the longitudinal PSF of $6.5 \mathrm{~mm}$ achieved with dynamic aberration correction, whereas the FWHM is $12.7 \mathrm{~mm}$ for the uncorrected case.

The most significant result of correction is exemplified by the un-normalized longitudinal PSF. Whereas the normalized PSF shows the decrease in FWHM due to the combination of

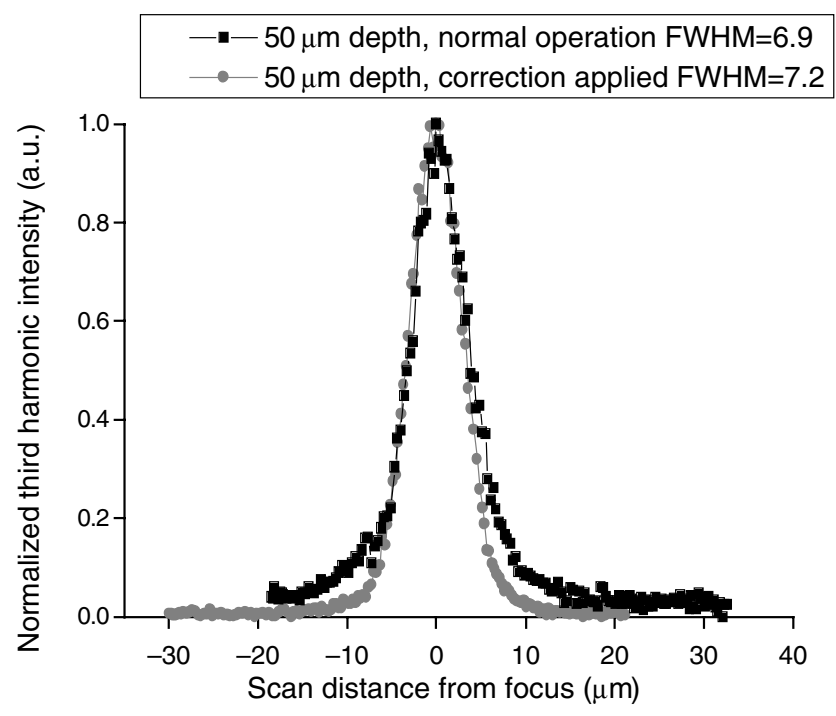

(a) spherical bias and DM correction, in contrast to operation with no bias and no correction, the un-normalized longitudinal PSF shows a remarkable improvement in third harmonic intensity that results from compensating the spherical aberration, which redirects the energy from the wings into the centre of the beam in the focal plane when focusing deep into a specimen with refractive index mismatch. Figure 10(a) shows the normalized PSF decrease in FWHM from $14.6 \mathrm{~mm}$ to $8.1 \mathrm{~mm}$ with adaptive correction at a focus depth of $450 \mathrm{~mm}$; the un-normalized curves (Fig. 10(b)) show a dramatic increase in the THG intensity at the focal plane.

\section{Conclusions}

We have demonstrated adaptive correction of depth-induced spherical aberrations in a Coumarin-water solution by a membrane deformable mirror. This was achieved by two methods, both utilizing a stationary bias. Using a spherical bias the depth scanning range was increased from $150 \mathrm{~mm}$ to $600 \mathrm{~mm}$, with negligible loss of energy due to insertion of the adaptive correction system. Using a defocus bias the depth scanning was increased from $150 \mathrm{~mm}$ to $800 \mathrm{~mm}$, but with $50 \%$ loss in energy due to insertion of the correction system.

\section{Acknowledgements}

The project has been funded in whole or in part with Federal Funds from the National Cancer Institute, National Institutes of Health, under contract \#NOI-CO-97111, and by the National Science Foundation through the Center for Ultrafast Optical Science under STC PHY 890108.

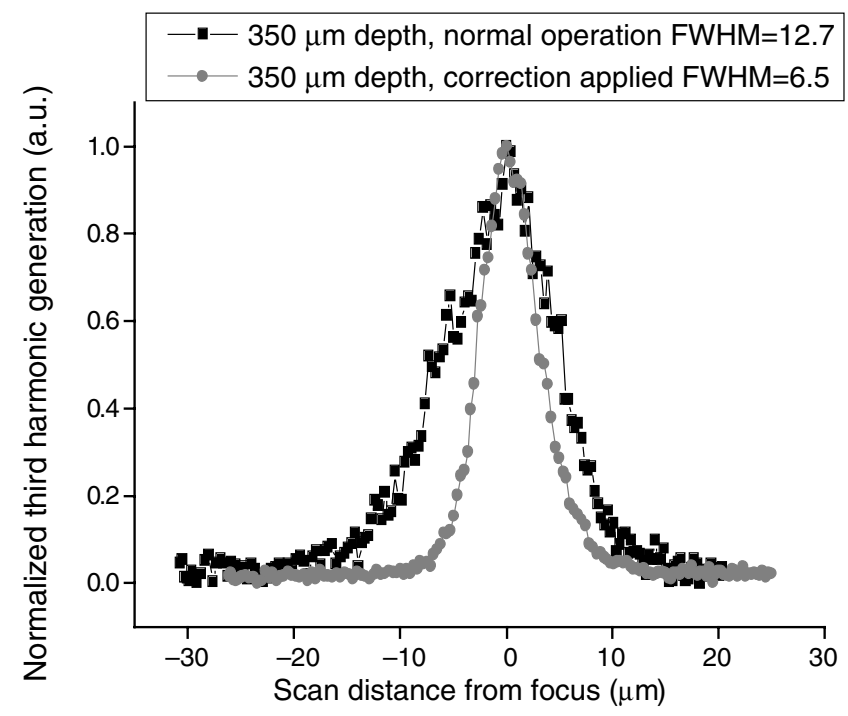

(b)

Fig. 9. The normalized longitudinal point spread shows significant improvement with correction at $350 \mathrm{~mm}$, with no adverse effects at $50 \mathrm{~mm}$. 


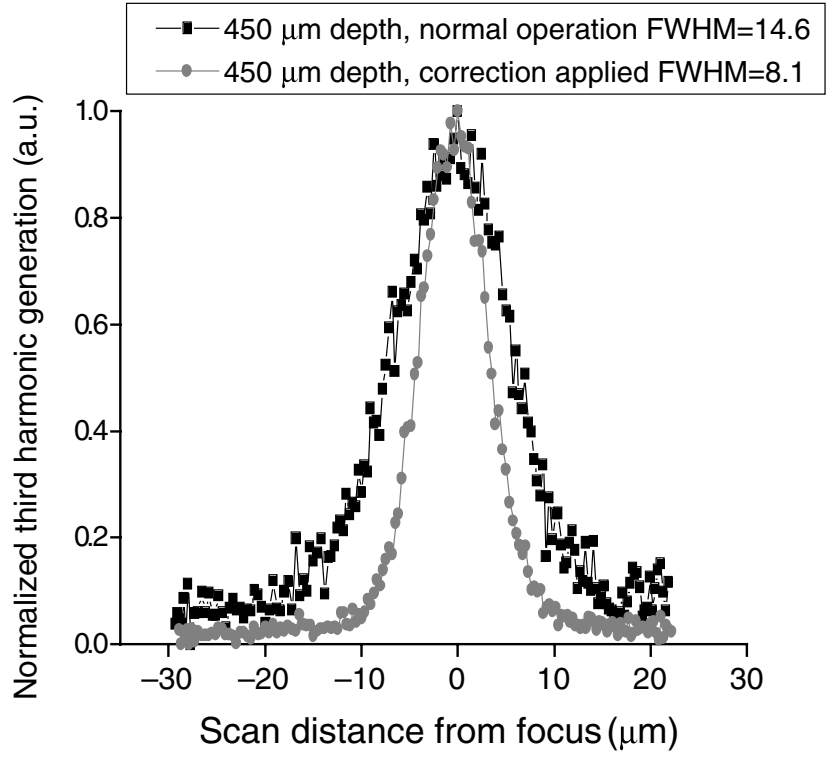

(a)

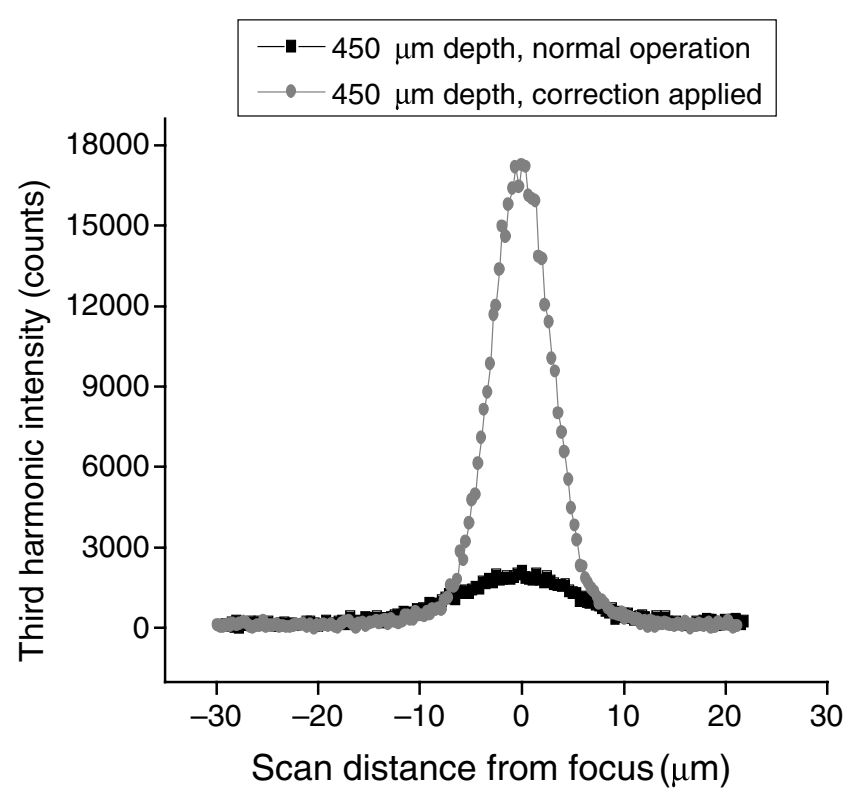

(b)

Fig. 10. Normalized longitudinal point spread for focusing depth of $450 \mathrm{~mm}$ for both corrected and uncorrected showing increase in resolution due to correction, as well as unnormalized showing increase in intensity due to correction.

\section{References}

Albert, 0., Sherman, L., Wovin, G., Mourou, G. \& Norris, T. (2000) Smart microscope: an adaptive optics learning system for aberration correction in multiphoton confocal microscopy. Opt. Lett. 25 , $52-54$

Booth, M.J., Neil, M.A.A. \& Wilson, T. (1998) Aberation correction for confocal imaging in refractive-index-mismatched media. J. Microsc. 192, 90-98.

Booth, M.J. \& Wilson, T. (2000) Strategies for the compensation of specimen induced spherical aberration in confocal microscopy of skin. J. Microsc. 200, 68-74.

Born, M. \& Wolf, E. (1980) Principles of Optics, 6th edn. Cambridge University Press, Cambridge.

Denk, W., Strickler, J. \& Webb, W. (1990) Two-photon laser scanning fluorescence microscopy. Science, 248, 73-76.

Hell, S.W., Rainer, G., Cremer, C. \& Stelzer, E.H.K. (1993) Aberrations in confocal fluorescence microscopy induced by mismatches in refractive index. J. Microsc. 169, 391-405.

Hellmuth, T., Seidel, R. \& Seigal, A. (1988) Spherical aberration in confocal microscopy. Proc. SPIE, 1028, 28-32.

Neil, M.A.A., Juškaitis, R., Booth, M.J., Wilson, T., Tanaka, T. \& Kawata, S. (2000) Adaptive aberration correction in a two-photon microscope. J. Microsc. 200, 105-108.
Primot, J. \& Sogno, L. (1995) Achromatic three-wave (or more) lateral shearing interferometer. J. Opt. Soc. Am. A, 12, 2679-2685.

Sheppard, C.J.R. \& Gu, M. (1991) Aberration compensation in confocal microscopy. App. Opt. 30, 3563-3568.

Török, R., Hewlett, S.J. \& Varga, P. (1997) The role of specimen-induced spherical aberration in confocal microscopy. J. Microsc. 188, 158-172.

Török, P., Varga, P., Laezik, Z. \& Booker, G.R. (1995) Electromagnetic diffraction of light focused through a planar interface between materials of mismatched refractive indices: an integral representation. J. Opt. Soc. Am. A, 12,325-332.

Tsang, T. (1995) Optical third-harmonic generation at interfaces. Phys. Rev. A, 52, 4116-4125.

Tyson, R. (1991) Principles of Adaptive Optics. Academic Press, Boston.

Vdovin, G., Middelhoek, S. \& Sarro, P. (1997) Technology and applications of micromachined silicon adaptive mirrors. Opt. Eng. 350, 1382-1390.

Wan, D.-S., Rajadhyaksha, M. \& Webb, R.H. (2000) Analysis of spherical aberration of a water immersion objective: application to specimens with refractive indices 1.33-1.40. J. Microsc. 197, 274-284.

Wilson, T. \& Sheppard, C.J.R. (1984) Theory and Practice of Scanning Optical Microscopy. Academic Press, London.

Zhu, L., Sun, P.-C., Bartsch, D.-U., Freeman, W.R. \& Fainman, Y. (1999) Wave-front generation of Zernike polynomial modes with a micromachined membrane deformable mirror. App. Opt. 38, 60196026. 